\title{
Mauvaise nouvelle pour le climat et les peuples de l'Amazonie équatorienne : l'abandon du projet Yasuni-ITT de gel du pétrole en terre
}

\author{
Michel Damian \\ Économiste, Université de Grenoble, PACTE, EDDEN, CNRS, 38040 Grenoble, France
}

Mots-clés :

Yasuni-ITT ; économie écologique ;

post-extractivisme ; OPEP ; changement climatique

\begin{abstract}
Résumé - Ce texte présente la proposition Daly-Correa de taxe et de compensation internationales pour un gel de l'exploitation pétrolière en Amazonie équatorienne - initiative Yasuni-ITT -, jusqu'à son abandon par le Président de l'Équateur, Rafael Correa, le 15 août 2013. Les enjeux concernent le développement des peuples autochtones, la politique des pays exportateurs de pétrole, la gouvernance internationale, les négociations climatiques et le maintien du carbone en terre, les luttes internes pour le Buen Vivir, mais également l'économie politique de chacune de ces questions et, en particulier, les positions et engagements d'économistes écologiques. Le texte suggère que la diplomatie française pourrait mettre le projet Yasuni-ITT - ainsi que la proposition Daly-Correa de taxation du pétrole exporté, et il conviendrait d'y ajouter des mesures pour le charbon - à l'agenda de la Conférence climatique qui se tiendra à Paris à la fin de l'année 2015. On peut douter que ce type de projet de compensation devienne un jour réalité. Il faut cependant en parler à Paris en 2015, pour ne pas évacuer un double défi : 1) celui du maintien d'une partie du carbone en terre et, 2) celui du « développement » - quelle que soit la définition que l'on en donne, sans retomber dans les naïvetés développementistes respectueux des communautés et des populations. Les conflits et négociations contemporains - c'est aussi vrai pour le climat - se rapprochent des sociétés et des acteurs sociaux, qui en deviennent les principaux protagonistes ; il est nécessaire d'intégrer ces acteurs extra-étatiques, fussent-ils localisés au cœur de l'Amazonie équatorienne.
\end{abstract}

Keywords:

Abstract - Bad news for the climate and the indigenous people of equatorial Amazon: The demise of Yasuni-ITT; ecological economics; post-extractivism; OPEC; climate change the Yasuni-ITT Initiative to keep oil in the ground. This article presents the Daly-Correa proposal concerning taxation and international compensations to stop oil exploitation in the Equatorial Amazon The Yasuni-ITT Initiative - before President Rafael Correa of Equator dropped it last August 15. Highly sensitive issues are involved: the development of autochthonous peoples, policies of oil exporting countries, international governance, climate negotiations on keeping carbon underground and the internal fights around the Buen Vivir. Also at stake is the political economy of all these issues, specially the positions and commitments of ecological economists. The author suggests that French diplomacy could include the Yasuni-ITT initiative in the Climate Conference agenda that will convene in Paris at end of 2015, adding to it the Daly-Correa proposition to tax exported oil and some policy measures concerning carbon. There is some scepticism about the feasibility and realism of this kind of international compensations, but they need to be discussed in Paris. It is important not to give up a double challenge: 1) keeping some quantity of carbon in the subsoil, 2) the issue of "development" - whatever the definition and without adopting developmentalist naïvetés - fully respectful of communities and populations. It is true also for climate that contemporary conflicts and negotiations move closer to societies and social actors who are becoming crucial protagonists. These extra-state actors must be incorporated even if they are buried in the heart of Equatorial Amazon.

Auteur correspondant : michel.damian@upmf-grenoble.fr 
L'initiative Yasuni-ITT est un projet inédit de préservation de l'environnement et de défense des peuples autochtones en Amazonie équatorienne, dans le parc Yasuni, précisément dans la zone Ishpingo-TambocochaTiputini, d'où le sigle ITT. La construction progressive de ce projet, aux enjeux multiples, remonte au milieu de la décennie 2000. Son originalité consistait à laisser sous terre environ $20 \%$ des réserves pétrolières de l'Équateur, afin de protéger un parc naturel possédant un des plus hauts niveaux de biodiversité au monde et abritant des peuples «non contactés », « en isolement volontaire ». En échange du maintien d'une partie de son stock de carbone en terre, et pour assurer un développement plus écologique de cette région, l'Équateur demandait à la communauté internationale - au nom du principe de coresponsabilité dans les problèmes environnementaux globaux - une compensation à hauteur de $50 \%$ des revenus qu'il aurait pu tirer de l'exploitation de ce pétrole. Un fonds fiduciaire, géré par le Programme des Nations unies pour le développement (PNUD), pour collecter et distribuer les contributions internationales, avait même été créé en août 2010. Des promesses de dons, en provenance en particulier d'États et de régions d'Europe, avaient été faites.

Convient-il d'en parler au passé ? Ce projet a été abandonné, par un décret - Decreto Ejecutivo N. 7 - signé par le Président de l'Équateur, Rafael Correa, le 15 août 2013. Voici l'histoire, dans ses grandes lignes. Les enjeux concernent le développement des peuples autochtones, la politique des pays exportateurs de pétrole, la gouvernance internationale, la politique climatique et le maintien du carbone en terre, les luttes politiques internes pour le Buen Vivir (la satisfaction des besoins fondamentaux des êtres humains, en vivant en harmonie avec la nature), mais également l'économie politique de chacune de ces questions.

\section{Pour une taxation du pétrole exporté}

Les analyses d'économistes écologiques sont à la source des fondements théoriques du projet Yasuni-ITT, et tout d'abord celles de Herman Daly, professeur émérite à $l^{\prime}$ Université du Maryland, aux États-Unis. Il est la figure marquante du courant "Économie écologique » (avec, en particulier, Joan Martinez-Alier; tous deux sont des cofondateurs de la revue Ecological Economics). Lors d'une conférence donnée à Vienne, au siège de l'Organisation des pays exportateurs de pétrole (OPEP), en 2001, il a proposé que cette organisation prélève une taxe de quelques pour cents sur chaque baril de pétrole exporté pour alimenter un fonds destiné à combattre les changements climatiques et soutenir les politiques de soutenabilité des pays en développement (Daly, 2001).
L'argumentation était la suivante. Le développement soutenable a pour objectif de sortir de ce que Daly appelle « la croissance non économique », c'est-à-dire la croissance du PIB, qui détruit les ressources naturelles et accroît les inégalités, pour aller vers un progrès plus qualitatif qui s'éloigne de la seule expansion quantitative (Daly, 2002). L'OPEP, déclarait-il, a un rôle à jouer, et pour le climat et pour un développement plus soutenable des pays pauvres. Sa thèse, sur laquelle il insiste, est que l'OPEP a un pouvoir de monopole, au moins relatif, qui peut lui permettre de prélever une taxe sur le pétrole exporté pour la redistribuer au bénéfice des pays pauvres.

Daly concluait avec retenue, mais en soulignant fortement, que l'OPEP lui apparaissait comme l'institution la plus à même de minimiser les flux de throughput (les prélèvements sur les stocks de carbone en terre et leurs rejets dans les puits à déchets) : « Maybe the whole idea is just a utopian speculation. [...] If sustainability is to be more than an empty word we have to evolve mechanisms for constraining throughput flows within environmental source and sink capacities. Petroleum is the logical place to begin. And OPEC is the major institution in a position to influence the global throughput of petroleum. » (p. 9).

Joan Martinez-Alier est à l'origine de la translation entre la proposition de Herman Daly et la politique écologique, en l'occurrence en Équateur.

\section{De l'économie écologique à la politique écologique}

Joan Martinez-Alier, professeur à l'Université autonome de Barcelone, est un des grands théoriciens - et représentants engagés -, non seulement de la décroissance, mais également de la dette écologique et du développement écologiquement inégal, de l'environnementalisme des pauvres et de la justice environnementale (Martinez-Alier, 2002, 2011 ; Cabeza-Gutés, MartinezAlier, 2001).

C'est Martinez-Alier (par ailleurs proche du mouvement équatorien Acción Ecológica, à l'origine, en 2006, de l'initiative Yasuni-ITT) qui a présenté, début 2007, ce projet de taxe à Fander Falconi, ministre du Plan, qui l'a lui-même soumis au Président Correa (Daly, 2012¹). Rafael Correa et son gouvernement, malgré des controverses et divergences fortes, ont par la suite toujours

\footnotetext{
1 Pour une présentation documentée de l'initiative YasuniITT, cf. Le Quang M. (2010), Martin (2011). Pour une approche plus en termes d'économie de l'énergie, $c f$. Larrea, Wanars (2009), Rival (2010), Escribano (2013). Sur la biodiversité de cette zone géographique, la plus remarquable en Amérique du Sud et peut-être au plan mondial, $c f$. Finer et al. (2009), Bass et al. (2010).
} 
défendu l'idée d'une telle taxation du pétrole à la source, susceptible de contribuer à un fonds financier pour la compensation du carbone maintenu en terre.

Le Président équatorien évoque pour la première fois publiquement l'idée lors d'un sommet de l'OPEP, tenu à Ryad le 18 novembre 2007, lors de la réintégration officielle de son pays au sein de l'organisation (l'Équateur, le plus petit des producteurs de l'OPEP, était sorti de l'organisation vingt-cinq ans auparavant). En décembre 2010, lors d'une réunion à Quito, il appelle à nouveau les pays membres de l'OPEP à soutenir l'idée d'une taxation des exportations pétrolières. En février 2012, toujours à Quito, le projet est réitéré lors d'une réunion des ministres de l'Environnement d'Amérique Latine et de la Caraïbe. Enfin, la proposition est explicitement avancée en octobre 2012, lors d'un sommet des pays arabes et d'Amérique du Sud tenu au Pérou, et auquel participaient les chefs d'État et ministres de l'Énergie de neuf des douze membres de l'OPEP.

Il était attendu que la proposition - connue sous la dénomination « taxe Daly-Correa » - soit officiellement annoncée et défendue lors de la Conférence sur le climat à Doha du 27 novembre au 7 décembre 2012.

\section{Disparue dans les sables du Qatar}

La proposition Daly-Correa a à peine été évoquée à Doha, dans quelque corridor climatisé du premier émetteur de $\mathrm{CO}_{2}$ par habitant de la planète, sans aucun écho dans les comptes rendus de la conférence. Selon Tarcisio Granizo, ministre équatorien du Patrimoine, qui dirigeait la délégation de son pays, le projet de taxe ne pouvait pas être présenté uniquement par un seul des pays producteurs de pétrole : «debería ser presentada por la OPEP » (EFEverde, 2012). Abdullah bin Hamad alAttiyah, le ministre qatari de l'Énergie et président de la Conférence de Doha, aurait cependant réagi, aux dires des diplomates équatoriens, « very positively » à la proposition, au point d'envisager de recommander qu'elle soit examinée lors de la réunion du conseil de l'OPEP prévue le 12 décembre 2012 à Vienne (Fineren, 2012).

Lors de ce conseil, l'OPEP a vu une nouvelle fois monter les tensions entre l'Iran et l'Arabie saoudite, ses membres n'ont pas réussi à se mettre d'accord sur le nom de son nouveau secrétaire général (le précédent a été reconduit), et le partage des quotas de production de l'organisation est toujours, avec le retour de l'Irak, très sensible. On pouvait donc être tout à fait sceptique sur la volonté et la capacité de l'OPEP à s'engager dans une taxation du pétrole au profit des politiques climatiques et de soutenabilité des pays en développement les moins avancés ou les plus vulnérables. Ce 12 décembre, depuis Vienne, le constat de Wilson Pástor-Morris, ministre équatorien des Ressources naturelles, était amer : " no está en el orden del día de esta sesión. » (El Universo, 2012).

L'espoir de la diplomatie équatorienne était que la question figure à l'ordre du jour du prochain conseil de l'OPEP, fin mai 2013 à Vienne. Cela n'a pas été le cas. Le projet Daly-Correa de taxe sur le pétrole exporté est enterré, pour longtemps au sein de l'OPEP. L'opposition des pays producteurs de pétrole à des mesures contraignantes pour réduire les émissions de $\mathrm{CO}_{2}$ ne date cependant pas de 2012 ou 2013 ; elle est ancienne, constitutive de la politique climatique multilatérale.

\section{Le barrage des pays producteurs de pétrole}

Lors de la première Conférence ministérielle sur la pollution atmosphérique et le changement climatique, tenue à Noordwijk, aux Pays-Bas, en novembre 1989, le prince Fahad Bin Abdullah Al Saud - aujourd'hui roi d'Arabie saoudite - caractérisait le réchauffement climatique comme " a life or death issue for considerable areas of the earth », reconnaissant qu'il n'y avait aucune controverse («no argument ») sur le fait que le responsable principal (the "main culprit») du réchauffement global était le dioxyde de carbone, et qu'il était nécessaire de stabiliser et réduire les émissions de gaz à effet de serre (cité in Bodansky, 1993, p. 467, note 100). Le prince n'avait visiblement pas encore saisi toutes les implications d'une politique conséquente de lutte contre les émissions de gaz à effet de serre, en particulier pour son pays.

Au printemps 1991, dès les premières négociations pour la rédaction de la Convention-cadre des Nations unies sur les changements climatiques (signée à Rio de Janeiro en juin 1992), l'Arabie saoudite, le Koweit, et avec eux tous les pays producteurs de pétrole, vont s'opposer aux propositions susceptibles de réglementer et limiter les émissions en provenance des sources de carbone : pas de taxe, aucune évocation de la mise en œuvre de standards d'efficacité énergétique, aucune référence aux sources d'énergies renouvelables. L'architecture climatique internationale, issue de la Convention-cadre en 1992, puis du protocole de Kyoto en 1997, se fixera comme objectif de réduire les "émissions" pour un panier amalgamant six gaz à effet de serre, mais cet objectif est en quelque sorte abstrait - et c'est la grande différence avec le protocole de Montréal pour limiter les substances qui appauvrissent la couche d'ozone -, puisque les conditions matérielles et productives de cette réduction des émissions ne sont pas spécifiées. Les revenus pétroliers étaient en jeu.

Les producteurs de pétrole obtiendront, au titre de l'article 4 de la Convention-cadre sur les changements 
climatiques (et de l'article 2 du protocole de Kyoto), le droit à compensation de leurs revenus pétroliers au cas où la réussite d'une politique de limitation des émissions carbonées viendrait à les diminuer. Pour faire bonne mesure, toujours au titre de ces articles, les pays producteurs de charbon bénéficient d'un droit identique à compensation. Il a fallu, en 1992, ce compromis inouï pour que les pays producteurs de pétrole acceptent finalement d'adhérer à la Convention (Dessai, 2004, pp. 20-21). Un compromis qui est passé presque inaperçu, en tout cas qui a été très peu commenté. Lors de chaque réunion relative au climat, l'Arabie saoudite ne manque, elle, jamais de rappeler ce droit à compensation.

Cette position réactive, de barrage, n'est pas seulement celle des pays pétroliers. La quasi-totalité des pays du G77 plus la Chine, et, en particulier, les grands pays émergents, s'opposent, tous, à toute tentative de mesures commerciales unilatérales pour réduire les émissions. Voici ce qu'exigeaient le Brésil, l'Afrique du Sud, l'Inde et la Chine (le groupe dit BASIC), en quelque sorte leur "contribution » pour la Conférence climatique de Copenhague en 2009: «Parties shall not resort to any form of unilateral measures, including fiscal and nonfiscal border measures, against goods and services imported from other Parties, in particular from developing country Parties, on grounds of stabilization and mitigation of climate change. » (cité in Barrett, 2010, p. 20).

Une fiscalité «climat » sur le pétrole importé ou sur les biens "carbonés » aux frontières est un casus belli pour le Sud dans son ensemble. Le dernier projet de loi pour lutter contre les changements climatiques, soumis au Congrès américain en 2009 (aucun des nombreux projets présentés ne réussit à passer le double obstacle de la Chambre des représentants et du Sénat), proposait d'instaurer un marché des permis d'émissions (système capand-trade) au niveau de l'État fédéral, pour donner un prix au carbone. Le projet de loi ouvrait la possibilité de taxer les importations de produits intensifs en $\mathrm{CO}_{2}$ en provenance de pays qui n'auraient pas adopté des mesures similaires pour réduire les émissions. La réaction du South Centre fut immédiate : " This would violate the provisions of Article 4 [de la Convention-cadre sur les changements climatiques], which exempts developing countries from uncompensated mitigation actions involving incremental costs. » (Dasgupta, 2009, p. 3). Comment faire?

\section{Una global hipocresia}

L'initiative Yasuni-ITT était remarquable à un double titre. Elle se singularisait par ses aspects sociaux, elle visait à protéger non seulement les forêts mais aussi les populations et leur lien avec la nature. Elle initiait des réseaux et des mécanismes, à la fois locaux et mondiaux, de gouvernance entre une myriade d'acteurs, pour s'orienter en direction d'un développement national tournant le dos à l'extractivisme et à la rente pétrolière (Martin, 2011).

La clé du projet : la compensation. Pour le maintien en terre d'environ 900 millions de barils de pétrole, l'Équateur espérait obtenir en compensation la moitié de ce qu'aurait pu lui rapporter l'exploitation, soit 350 millions de dollars par an sur une période de treize ans ${ }^{2}$. Des États et des régions, essentiellement d'Europe, mais aussi des firmes multinationales, s'étaient engagés, par des dons déjà versés ou des promesses, à contribuer au financement $\mathrm{du}$ fonds géré par le $\mathrm{PNUD}^{3}$. Dons et promesses n'étaient cependant pas en ligne avec ce qu'escomptait l'Équateur. Au printemps 2013, semble-til, un peu plus de 10 millions de dollars étaient déjà versés, et les promesses avoisinaient 100 millions de dollars, ce qui était très loin du montant attendu chaque année, durant 13 ans.

Les grandes puissances ont toujours signifié, du moins jusqu'à présent, leur refus ou réticence extrême à s'engager dans quelque forme de « compensation » que ce soit (qui s'apparente, selon elles, à du « chantage » écologique ${ }^{4}$. Et l'engrenage dans la compensation conduit à des chiffres, comment dire, "irréalistes »: les réserves mondiales de pétrole, techniquement et économiquement exploitables, sont évaluées aujourd'hui à un peu moins de 1500 milliards de barils ; s'il fallait compenser le gel en terre de ces réserves mondiales aux conditions équatoriennes, cela représenterait, "en gros», une somme annuelle de 500 milliards de dollars par an durant treize ans.

Il y aurait pourtant une très bonne raison de considérer avec la plus grande attention l'initiative Yasuni-ITT et l'hypothèse de la compensation. S'il s'agit bien de limiter les émissions de carbone, alors que les combustibles fossiles représenteront encore au moins $70 \%$ de la

\footnotetext{
2 Pour une évaluation plus élaborée du pricing des stocks et puits de carbone des champs pétrolifères ITT, dans une perspective économie écologique, $c f$. Rival (2010).

3 À notre connaissance, aucun pays producteur de pétrole n'avait versé ou promis quoi que ce soit.

4 Le projet avait beaucoup d'ennemis, à l'intérieur de l'Équateur - le lobby pétrolier était bien sûr contre, y compris la compagnie pétrolière nationale -, mais aussi à l'extérieur, avec, en Europe, le ministre fédéral de la Coopération économique du gouvernement allemand, Dirk Niebel, fortement opposé à la «compensation » : « he insists, that "Germany will not contribute to a fund that is based on the philosophy of 'payment for non-action"'. Responding to intense pressure to assist in Yasuni, he did trickle down some euro (24 millions is nothing to sneeze at), but instead of being part of a project to leave the oil under the soil, he's only interested in market-oriented projects like REDD. » (Bond, in Martinez-Alier et al., 2013).
} 
production énergétique au milieu de ce siècle, pourquoi ne pas commencer avec le gel de l'exploitation pétrolière initialement prévu dans le cadre de ce projet ? La compensation financière pour ce maintien de carbone en terre ne pourrait-elle pas, d'ailleurs, figurer à l'ordre du jour de prochaines négociations climatiques multilatérales ? En effet, si les pays producteurs de pétrole ont pu se voir reconnaître, à Rio de Janeiro en 1992, un droit à compensation au cas où la réussite de la politique climatique viendrait à diminuer la consommation de pétrole et donc leurs revenus, pourquoi l'Équateur ne pourrait-il se voir reconnaître un droit identique à compensation, dans ce cas pour une perte de revenus due à un maintien - volontaire et collectivement assumé -, de carbone en terre?

Pas de taxe, même très modeste, sur les exportations de pétrole pour financer, au moins pour une partie, la survie soutenable de peuples autochtones, de populations «non contactées », ainsi que la préservation de la biodiversité en Amazonie équatorienne. Échec de la tentative de réunir les sommes escomptées dans le cadre du fonds financier géré par le PNUD. « Una global hipocresia », se lamentait le 15 août 2013 le Président Rafael Correa (El Universo, 2013).

\section{Maintenir le carbone en terre pour préserver le climat?}

Les mouvements de résistance à l'exploitation pétrolière dans les pays tropicaux, et en particulier dans les territoires indigènes et aires protégées, sont déjà anciens $s^{5}$. La question du maintien du carbone en terre est en revanche toute récente. Elle est au croisement de travaux d'économistes, de scientifiques et spécialistes du climat, ainsi que de think tanks et campagnes activistes.

Au sein du monde académique et des économistes, la publication princeps revient à Hans-Werner Sinn, à l'origine, en 2008, du thème - controversé - du green paradox (imposer une taxation du carbone élevée et à taux croissant aux combustibles fossiles - pour contraindre au maintien de carbone en terre - conduirait leurs possesseurs à en accélérer l'exploitation, avec en résultat un emballement des émissions de $\mathrm{CO}_{2}$ ) (Sinn, 2008, 2012). La thèse d'ensemble de Sinn est que l'économie du changement climatique et les négociations multilatérales ont jusqu'à présent visé exclusivement l'atténuation des émissions, le versant «demande » du problème. Or le

\footnotetext{
5 Avec, en particulier, l'organisation Oilwatch, créée en 1995 en Équateur, liée à des mouvements situés au Nigéria, en Afrique du Sud, au Cameroun, Gabon, Thaïlande, Sri Lanka, Timor Oriental, Mexique, Guatemala, Pérou, Colombie et Brésil. Sur les perspectives qu'ouvrent ces mouvements, ainsi que l'initiative Yasuni-ITT, $c f$. Martinez-Alier (2013).
}

versant « offre », la question du carbone en terre, susceptible d'être extrait et émis dans l'atmosphère, avec un stock de combustibles fossiles qui n'attend que son exploitation, est encore plus préoccupant : «the overall stocks of resources for oil, gas, and coal in terms of carbon content range from 3,967 to $5,579 \mathrm{Gt}$. If $45 \%$ of the lower of these two quantities enters the atmosphere, the stock of oxidized carbon existing there would increase from today's $809 \mathrm{Gt}$ to $2,594 \mathrm{Gt}$, i.e. by $221 \%$. The concentration of carbon dioxide in the atmosphere would accordingly increase from $380 \mathrm{ppm}$ to about 1,220 ppm, far more than any model projections thus far have dared to predict. » (Sinn, 2008, p. 365).

Il y a donc ce que l'on appelle aujourd'hui du unburnable fuel (The Economist, 2013) et du unburnable carbon (Carbon Tracker, 2013). À l'origine de la problématique, il y a les travaux, cette fois de scientifiques du climat, sur le carbon budget, mesuré en tonnes de $\mathrm{CO}_{2}$, dont disposerait encore l'humanité pour ne pas dépasser un seuil de réchauffement de $2{ }^{\circ} \mathrm{C}^{6}$. Or ce budget est très limité, ce qui implique de réduire rapidement et fortement les émissions, avec l'absolue nécessité de ne pas exploiter la totalité des combustibles fossiles encore disponibles.

Si l'objectif est bien de ne pas dépasser un réchauffement de $2{ }^{\circ} \mathrm{C}, 60$ à $80 \%$ des réserves identifiées, et aujourd'hui économiquement exploitables, devraient rester en terre. Il y a donc une «bulle carbone » qui pèse sur les milliards de dollars investis dans les entreprises qui exploitent pétrole et autres combustibles fossiles (Carbon Traker, 2013 ; Stern, 2011). L'urgence serait alors de désinvestir de ces entreprises, puisque la limitation des volumes extraits et le gel d'une partie de leurs réserves, avec la reconnaissance d'un unburnable carbon, dévaloriseraient leurs actifs ${ }^{7}$. Une carbon divestment campaign est en cours, animée par Bill McKibben et l'organisation 350.org.

Il y a près de quatre décennies, Alvin Weinberg, physicien nucléaire et scientifique célèbre pour ses prises de position, soutenait déjà qu'il faudrait peut-être un jour limiter l'exploitation des combustibles fossiles : "we may have to limit our burning of fossil fuel because of a possible $\mathrm{CO}\left[\mathrm{CO}_{2}\right]$ catastrophe. » (Weinberg, 1977). D'autres, quelques rares économistes ou scientifiques, étaient, eux, sans espoir sur la possibilité de restreindre l'extraction de pétrole et de charbon. En 1983, Thomas

\footnotetext{
6 L'article, qui a le plus influencé ce débat (il est déjà cité quelque 800 fois dans la littérature académique), a été publié dans la revue Nature au printemps 2009, sous la direction de Malte Meinshausen (2009).

7 La capitalisation boursière des entreprises (publiques, privées, du Sud, du Nord) détentrices et exploitantes de pétrole et autres combustibles fossiles, s'élève actuellement (selon les chiffres de Carbon Tracker) à quelque 7 mille milliards de dollars.
} 


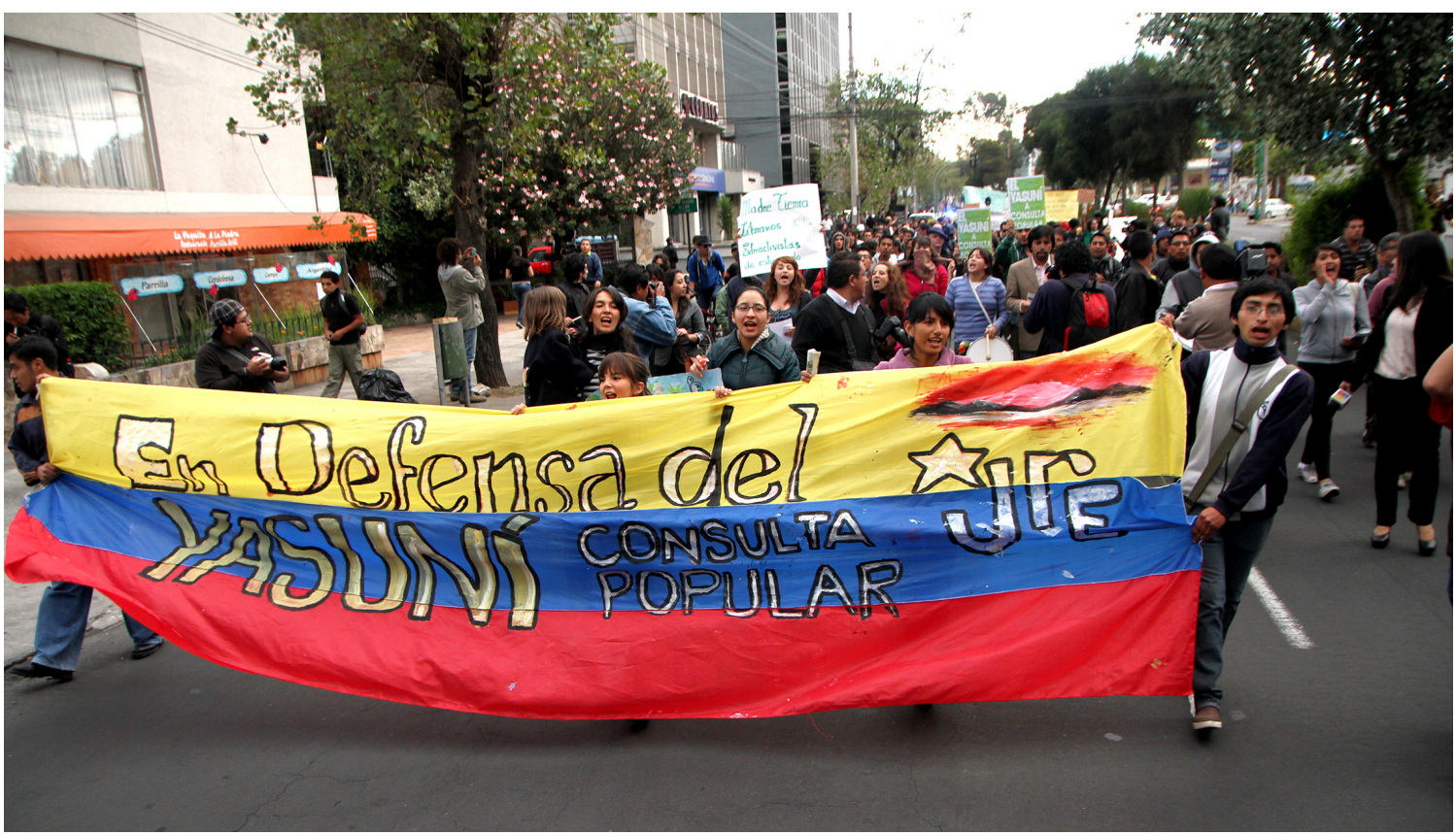

Manifestation à Quito le 5 septembre 2013. Des manifestants, opposés à la décision de mettre fin à l'initiative Yasuni-ITT, se sont regroupés devant la société pétrolière Petroamazonas (@ Luis Astudillo C./Agencia Andes).

Schelling, prix Nobel d'économie 2005, soutenait déjà que maintenir une partie des combustibles fossiles en terre était une perspective à peu près inenvisageable : «In the current state of affairs the likelihood is negligible that the three great possessors of the world's known coal reserve - the Soviet Union, the People's Republic of China, and the United States of America - will consort on an equitable and durable program for restricting the use of fossil fuels through the coming century » (1983, p. 481).

Le climatologue William Kellog, en 1987, était définitivement pessimiste, tranchant par la négative l'éventualité d'un accord multilatéral pour bannir ou réduire l'exploitation des combustibles fossiles : " Is there any international mechanism that could make such a drastic decision, and then enforce it on the world? The answer is, of course, a definite ' $\mathrm{No}^{\prime}$ » $(1987$, p. 125). Faut-il désespérer?

\section{Economista ecológico versus economista anti-ecológico}

L'abandon du projet Yasuni-ITT a aussi sa source dans des tensions internes aux forces sociales en Équateur. Il est en particulier emblématique des débats latino-américains, mais aussi équatoriens, sur le développement. À un extrême, les thèses récentes sur le new developmentalism, qui sont muettes sur les relations entre développement et environnement (le présupposé économique fondamental est qu'il y a une tendance à la surappréciation du taux de change) (Bresser-Pereira, 2006). À l'autre extrême, les thèses post-extractivistes, qui s'opposent aux politiques prédatrices sur les matières premières et l'environnement par les multinationales, et défendent un développement reconnaissant les droits des peuples autochtones et des populations, ainsi que les «droits de la nature» (qui sont inscrits dans la réforme de la constitution équatorienne de 2008). Alberto Acosta, théoricien du Buen Vivir, ministre équatorien de l'Énergie et des Mines en 2007, est un des leaders latino-américains de ce courant (Acosta, 2009). Il est également un penseur de la sortie du pétrole extorqué par les multinationales : le pétrole n'a pas contribué à développer le pays, et il a détérioré les conditions environnementales et sociales de la population amazonienne, il est donc urgent de penser à un développement post-pétrole (Acosta, 2000).

Alberto Acosta a toujours porté le projet Yasuni-ITT au plus haut niveau politique. Mais les affrontements au sommet de l'État ont été constants et, depuis son lancement, le projet se heurte à d'innombrables difficultés. Le Président Rafael Correa, lui, a toujours eu « un plan B», comme dit Martinez-Alier : si la communauté internationale ne contribue pas, ou pas suffisamment, à la compensation proposée, le pays ne pourra pas faire autrement pour son développement que d'exploiter le pétrole du bloc ITT du parc amazonien. L'économie de l'Équateur est en effet principalement basée sur la rente pétrolière, qui représente plus de $45 \%$ du budget de 
l'État; avec une politique de l'énergie et de l'exploitation pétrolière écartelée entre nationalisme, conservation, exploitation et des financements chinois pour à peu près tous les projets : «If something seems clear and consistent in Ecuador's energy strategy, it is the attraction of Chinese loans. » (Escribano, 2013, p. 158).

Dans cet environnement tendu, des élections à la Présidence de l'Équateur ont eu lieu en février 2013. Alberto Acosta, qui fait partie des figures emblématiques du pays, était l'un des candidats, contre Rafael Correa. Avec des craintes quant à l'avenir du projet, comme il l'exprimait au cours de la campagne électorale : "If Correa wins the ITT initiative will be dropped. » (Varas et al., 2013).

Joan Martinez-Alier a choisi : «Alberto Acosta es un economista ecológico y Rafael Correa es en la práctica un economista anti-ecológico » (Martinez-Alier, 2012a ; également 2012b). Mais c'est Rafael Correa qui est sorti vainqueur des élections présidentielles, avec $57 \%$ des suffrages.

\section{Conclusion}

L'abandon du projet Yasuni-ITT n'est pas une bonne nouvelle pour les peuples autochtones, pour le climat non plus. La politique climatique est en grande difficulté (Damian, 2012, 2013 ; Geden, 2013a, 2013b). Les négociations sont même un fiasco, si l'on s'en tient aux objectifs de réduction successivement proposés pour les décennies à venir (en termes de $2{ }^{\circ} \mathrm{C}$ de réchauffement à ne pas dépasser, ou de 50-80\% d'émissions en moins d'ici 2050) : on sait aujourd'hui qu'ils ne seront pas tenus. L'histoire du projet Yasuni-ITT n'est cependant pas terminée, il pourrait être à nouveau porté par les mouvements écologistes équatoriens et leurs soutiens internationaux ${ }^{8}$. La France s'était engagée à contribuer au fonds géré par le PNUD pour préserver les populations et l'environnement de cette partie de l'Amazonie. Une suggestion : la diplomatie française pourrait tout à fait mettre le projet Yasuni-ITT - ainsi que la proposition Daly-Correa de taxation du pétrole exporté, et il conviendrait d'y ajouter des mesures pour le charbon - à l'agenda de la Conférence climatique qui se tiendra à Paris à la fin de l'année 2015.

On peut douter que ce type de projets de compensation devienne un jour réalité. Il faut cependant en parler

\footnotetext{
8 Le 22 août 2013, au nom des mouvements sociaux et écologistes, le juriste et professeur Catedrático, Julio César Trujillo, a présenté une requête auprès de la Cour constitutionnelle pour annulation de l'exploitation des champs pétrolifères de la zone Yasuni-ITT, « por un tiempo indefinido ». L'objectif des mouvements politiques est aussi d'impulser une consultation populaire ou un référendum national (article 104 de la Constitution de 2008) contre cette exploitation (El Commercio, 2013).
}

à Paris en 2015, pour ne pas évacuer un double défi : 1) celui du maintien d'une partie du carbone en terre et, 2) celui du « développement » - quelle que soit la définition que l'on en donne, sans retomber dans les naïvetés développementistes - respectueux des communautés et des populations. Les conflits et négociations contemporains - c'est aussi vrai pour le climat - se rapprochent des sociétés et des acteurs sociaux, qui en deviennent les principaux protagonistes ; il est nécessaire $\mathrm{d}^{\prime}$ intégrer ces acteurs extra-étatiques, fussent-ils localisés au cœur de $l^{\prime}$ Amazonie équatorienne : «On voit [...] poindre une histoire infiniment plus dense, plus universelle, plus sociale et humaine, aussi tragique soit-elle ou restet-elle. » (Badie, 2013, p. 57).

\section{Remerciements}

Mes remerciements chaleureux à Joan Martinez-Alier et Angel de la Vega Navarro pour leurs précisions et commentaires, et à Vincent Plauchu pour sa relecture et ses conseils.

\section{Références}

Acosta, A. (Ed.), 2000. El Ecuador post petrolero, Quito, Acción Ecológica.

Acosta, A., 2009. La maldicion de la abundancia, Quito, Abya Yala.

Badie, B., 2013. Quand l'Histoire commence, Paris, CNRS Éditions.

Barrett, S., 2010. Climate change and international trade: Lessons on their linkage from international environmental agreements. Background paper, $2^{\text {nd }}$ Conference Climate change, trade and competitiveness: Issues for the WTO, $16^{\text {th }}$, $17^{\text {th }}$ and $18^{\text {th }}$ June, Geneva, The Graduate Institute, http:/ / www.wto.org/english/res_e/reser_e/climate_jun10_e/ background_paper6_e.pdf.

Bass, M.S., Finer, M., Jenkins, C.N., Kreft, H., CisnerosHeredia, D.F., et al., 2010. Global conservation significance of Ecuador's Yasuní National Park, PLoS ONE, 5, 1, 1-22.

Bodansky, D., 1993. The United Nations framework convention on climate change: A commentary, Yale Journal of International Law, 18, 451-558.

Bresser-Pereira, L.C., 2006. New developmentalism and conventional orthodoxy, Économie appliquée, 59, 3, 61-94.

Cabeza-Gutés, M., Martinez-Alier, J., 2001. L'échange écologiquement inégal, in Damian, M., Graz, J.-C. (Eds), Commerce international et développement soutenable, Paris, Economica, 159-185.

Carbon Tracker, 2013. Unburnable carbon 2013: Wasted capital and standard assets, Carbon Tracker/Grantham Research Institute on Climate Change and the Environment, London School of Economics and Political Science (LSE), London.

Daly, H.E., 2001. Sustainable development and OPEC. Invited paper for the conference OPEC and the global energy balance: Towards a sustainable energy future, Vienna, September. Le 
texte est reproduit in Daly, H.E., 2007. Ecological economics and sustainable development, Cheltenham, Edward Elgar.

Daly, H.E., 2002. Sustainable development: Definitions, principles, policies. Invited Address, World Bank, Washington DC, April 30. Le texte est reproduit in Keiner, M., 2006 (Ed.). The future of sustainability, Dordrecht, Springer.

Daly, H.E., 2012. The Daly-Correa tax: Background and explanation, The Daly News, Center for the Advancement of the Steady State Economy (CASSE), November 26, http:/ / dalynews.org/learn/blog/page/7/.

Damian, M., 2012. Repenser l'économie du changement climatique, Économie appliquée, 65, 2, 9-46.

Damian, M., 2013. La politique climatique change enfin de paradigme, Économie appliquée. À paraître.

Dasgupta, C., 2009. International trade and climate change, Policy Brief $N^{\circ} 16$, South Centre, Geneva, September, http:/ / www.southcentre.int/wp-content/uploads/2013/06/ PB16_rade-and-Climate-Change_EN.pdf.

Dessai, S., 2004. An analysis of the role of OPEC as a G77 member at the UNFCCC. Report for WWF, December, http:// wwf.panda.org/?17192/An-Analysis-of-the-Role-ofOPEC-as-a-G77-Member-at-the-UNFCCC.

EFEverde, 2012. Ecuador espera que la OPEP asuma su impuesto ambiental al petróleo, 12 décembre.

El Commercio, 2013. Políticos y activistas presentaron pregunta para consulta sobre Yasuní, 22 août.

El Universo, 2012. Ecuador pedirá que la OPEP trate el impuesto ambiental al petróleo, 12 décembre.

El Universo, 2013. Rafael Correa pone fin a la iniciativa Yasuní ITT, 15 août.

Escribano, G., 2013. Ecuador's energy policy mix: Development versus conservation and nationalism with Chinese loans, Energy Policy, 57, 152-159.

Finer, M., Vijay, V., Ponce, F., Jenkins, C.N., Kahn, T.R., 2009. Ecuador's Yasuní Biosphere Reserve: A brief modern history and conservation challenges, Environmental Research Letters, 4, 3, August 24.

Fineren, D., 2012. Ecuador sees support for OPEC climate levy plan, The Daily Star, December 8.

Geden, O., 2013a. Modifying the $2^{\circ} \mathrm{C}$ target: Climate policy objectives in the contested terrain of scientific policy advice, political preferences, and rising emissions. SWP research paper/ RP05, German Institute for International and Security Affairs, Berlin, June 5, http://www.swp-berlin.org/en/ publications/swp-research-papers/swp-research-paperdetail/article/climate_modifying_the_2_c_target.html.

Geden, O., 2013b. A new climate-policy paradigm, Project Syndicate, August 7, http://www.project-syndicate.org/ commentary/replacing-the-top-down-approach-tointernational-climate-policy-by-oliver-geden.

Kellog, W.K., 1987. Mankind's impact on climate: The evolution of an awareness, Climatic Change, 10, 2, 113-136.
Larrea, C., Warnars, L., 2009. Ecuador's Yasuni-ITT initiative: Avoiding emissions by keeping petroleum underground, Energy for Sustainable Development, 13, 3, 219-223.

Le Quang, M., 2010. Une innovation dans la lutte contre le réchauffement climatique: L'initiative Yasuni-ITT en Équateur, Mouvements, 19 septembre.

Martin, P.L., 2011. Payer pour préserver : La politique internationale de la proposition équatorienne Yasuní-ITT, International Development Policy | Revue internationale de politique de développement, 10 mai.

Martínez-Alier, J., 2002. The environmentalism of the poor: A study of ecological conflicts and valuation, Cheltenham, Edward Elgar.

Martínez-Alier, J., 2011. Justice environnementale et décroissance économique : L'alliance de deux mouvements, Écologie \& Politique, 41, 1, 125-141.

Martínez-Alier, J., 2012a. Commentarios, in Burbano de Lara, F., Acosta versus Correa, Diario HOY - Noticia de Ecuador, hoy.com.ec, 4 septembre.

Martínez-Alier, J., 2012b. Punto de separación, EcuadorLibreRed, 2 octobre.

Martínez-Alier, J., 2013. Yasunizing the world, Triple Crisis, May 10.

Martínez-Alier, J., Bassey, N., Bond, P., 2013. Yasuni ITT is dead. Blame President Correa, Environmental Justice Organisations, Liabilities and Trade (EJOLT), August 17, http:// www.ejolt.org/2013/08/yasuni-itt-is-dead-blame-president-correa/.

Meinshausen, M., Meinshausen, N., Hare, W., Raper, S.C.B., Frieler, K., Knutti, R., Frame, D.J., Allen, M.R., 2009. Greenhouse-gas emission targets for limiting global warming to $2^{\circ} \mathrm{C}$, Nature, $458,1158-1162$.

Rival, L., 2010. Ecuador's Yasuní initiative: The old and new values of petroleum, Ecological Economics, 70, 2, 358365.

Schelling, T.C., 1983. Climatic change: Implications for welfare and policy, in National Research Council, Changing climate: Report of the carbon dioxide assessment committee, Washington DC, National Academy Press, 449-482.

Sinn, H.W., 2008. Public policies against global warming: A supply side approach, International Tax and Public Finance, $15,4,360-394$.

Sinn, H.W., 2012. The green paradox, Cambridge, Mass, MIT Press.

Stern, N., 2011. A profound contradiction at the heart of climate change policy, Financial Times, December 8.

The Economist, 2013. Unburnable fuel, May 4.

Varas, E., Ribadeneira, M., Watts, J., 2013. Ecuador election: Rafael Correa set to win despite fossil fuel fears, theguardian.com, February 14.

Weinberg, A., 1977. Towards an acceptable nuclear future. International Conference Nuclear Power and its Fuel Cycle, International Atomic Energy Agency, Salzburg, May 5. 